Research Paper

\title{
Infiltrating Immune Cells in Gastric Cancer: A Novel Predicting Model for Prognosis
}

Wenjie Li ${ }^{1,2^{*}}$, Mengting Li ${ }^{1,2^{*}}$, Haizhou Wang ${ }^{1,2}$, Yanan Peng ${ }^{1,2}$, Shouquan Dong1,2, Yuanyuan Lu' ${ }^{1,2}$, Fan Wang ${ }^{1,2}$, Fei Xu ${ }^{1,2}$, Lan Liu ${ }^{1,2}$ and Qiu Zhao ${ }^{1,2}$

1. Department of Gastroenterology, Zhongnan Hospital of Wuhan University, Wuhan, China.

2. Hubei Clinical Center and Key Lab of Intestinal and Colorectal Diseases, Wuhan, China.

*These authors contributed equally to this study.

$\square$ Corresponding authors: Lan Liu, E-mail: 1liugi@whu.edu.cn \& Qiu Zhao, E-mail: qiuzhao@whu.edu.cn.

(c) The author(s). This is an open access article distributed under the terms of the Creative Commons Attribution License (https://creativecommons.org/licenses/by/4.0/). See http://ivyspring.com/terms for full terms and conditions.

Received: 2020.07.25; Accepted: 2020.11.01; Published: 2021.01.01

\begin{abstract}
Objective: Immune cells infiltrating has been proved to be associated with prognosis in gastric cancer (GC) by studies. This study aims to explore the prognosis value of infiltrating immune cells in gastric cancer.

Methods: In our study, the CIBERSORT algorithm was used to calculate the fraction of 22 tumor-infiltrating immune cells (TIIC) in 100 normal and 300 tumor samples from the GEO cohort and 30 normal and 344 tumor samples from the TCGA cohort. Univariate and multivariate Cox regression were used to construct an immune risk score model. Multivariate cox regression was also used to validate whether our risk score model could predict prognosis in GC independently. Furthermore, the model was validated in different patient subgroups to test its independence. $P<0.05$ was considered statistically significant.

Results: The results showed that the fraction of 3 immune cells increased in tumor tissues compared with normal tissues in both the GEO and TCGA cohort. Univariate cox regression analysis showed four cells significantly correlated with survival rate in GC $(P<0.05)$. The immune risk score model was constructed based on the four cells through multivariate cox regression and further validated. The $K M$ survival curve suggested that patients with high risk had poor prognosis than patients with low risk $(P<0.05)$. ROC curve indicated the model was reliable $(A \cup C=0.67$ in the GEO cohort, $A U C=0.65$ in the TCGA cohort). Furthermore, multivariate Cox regression showed the model was an independent factor for overall survival predicting in GC (hazard ratio $(\mathrm{HR})=2.35,95 \%$ confidence interval $(\mathrm{Cl})=1.63 \sim 3.40$ in the $\mathrm{GEO}$ cohort, $\mathrm{HR}=2.87,95 \% \mathrm{Cl}=$ 1.94 4.25 in the TCGA cohort). Finally, we validated the model in patient subgroups by the KM survival curve.

Conclusion: In summary, tumor-infiltrating immune cells play an essential role in GC progression and affect the outcome of GC patients. The immune risk score can predict overall survival for GC independently, and high immune risk score is associated with poor prognosis.
\end{abstract}

Key words: gastric cancer, TIICs, immune risk score model, prognosis, multivariate cox regression

\section{Introduction}

Human gastric cancer is common cancer and the third leading cause of cancer-related death based on the Global Cancer Statistics 2018 [1]. Gastric cancer is a heterogeneous disease, and the outcome may change significantly even for patients with similar clinical characteristics and treatment options [2,3]. The current method of staging by pathology and tumor-node-metastasis (TNM) staging system is critical for choosing appropriate treatment [4], but it is not enough for prognostication and risk stratification
$[2,3]$. Therefore, it is necessary to identify novel biomarkers providing high predicting values and raising the prognostication.

During the complex development of human tumors, six biological capabilities are recognized as the hallmarks of cancer [5]. Tumors recruit a large number of normal cells, including immune cells, vessels, and fibroblasts to constitute the tumor microenvironment in which immune cells can foster multiple biological capabilities [6]. It has been 
revealed that many solid tumors are associated with immune cell infiltration in the tumor immune microenvironment (TIME) which affects therapy efficacy and overall survival to no small extent [7-9]. A study revealed that high T-cell subsets density in gastric cancer was associated with prolonged survival [10]. What is more, it was thought that natural killer cells and dendritic cells were promising targets of immunity therapy for gastric cancer [11, 12]. Hence, research on immune cells is critical for patient stratification and therapy selection.

In this study, we used CIBERSORT to calculate the composition of infiltrating immune cells in Gastric cancer and investigate the correlation between immune cells and overall survival and stages. A risk score model was constructed by multivariate cox regression to predict the overall survival of Gastric cancer, and the model was validated using clinical characteristics by univariate cox regression and multivariate cox regression.

\section{Methods}

\section{Patient Cohort and Data Preparation}

The training cohort is the ACRG cohort included 300 patients from the Asian Cancer Research Group study (GEO, https://www.ncbi.nlm.nih.gov/geo/) (GSE66229), with Affymetrix Human Genome U133 Plus 2.0 Array expression series matrix files and GPL570 platform. The testing cohort contained 348 patients from the "TCGA-STAD" project and the corresponding level-3 gene expression data were obtained from the Genomic Data Commons (https:/ / portal.gdc.cancer.gov). The expression data was the HTSeq-FPKM type. For datasets in the GEO cohort, the robust multiarray average algorithm was applied for background correction and quartile normalization. For gene symbols with multiple probes, the average value was calculated as expression level. For both cohorts, only patients with available expression profiles, clinical, pathologic, and survival data were included for analyses.

\section{Evaluation of Infiltrated Immune Cells}

The CIBERSORT algorithm was used to calculate the proportion of immune cells in gastric tumor tissues and normal tissues, with reference to LM22 gene signature [13]. The CIBERSORT algorithm is an analytical tool, containing 547 marker genes' expression signature matrix for calculating the fractions of infiltrated immune cell composition. LM22 defines 22 subtypes of immune cells referring to the annotated gene signature matrix, downloaded from the CIBERSORT website portal (https:// cibersort.stanford.edu/).

The 22 immune cells contain two subtypes of B cells, seven subtypes of T cells, two subtypes of NK cells, three subtypes of Macrophages, Monocytes, Dendritic cells resting, Dendritic cells activated, Mast cells resting, Mast cells activated, Eosinophils and Neutrophils. Samples with CIBERSORT $p$-value $<0.05$ were kept for the following analysis.

\section{Identification of Immune Cells correlated with Clinical Information}

Only samples with CIBERSORT $p$-value $<0.05$ were selected for clinical analysis in both TCGA and GEO cohort. The correlations between immune cells and overall survival and tumor pathologic stages were analyzed after merging selected immune cell expression matrix with the clinical information matrix. The cells with $p$-value $<0.05$ were considered as statistically significant.

\section{Immune Risk Score Model Construction}

Univariate Cox proportional hazards regression was conducted in the training cohort (the GEO cohort) using the infiltrated immune cells, and cells with $p$-value $<0.5$ were included for subsequent analysis. "glmnet" R package [14] was used to construct the immune risk score model through multivariate Cox proportional hazards regression. Moreover, cells with $p$-value $<0.05$ were considered as independent prognostic indexes.

\section{Statistical Analysis}

R 4.0.1 and appropriate packages were used to conduct all statistical analyses. The infiltrations of 22 immune cells were assessed by Wilcox test. Correlations among different types of immune cells were analyzed by "corrplot" R package. Kaplan-Meier survival curve was analyzed by "survival" $R$ package and evaluated by log-rank test. The correlations between immune cells and pathologic stage and TNM stage were evaluated by Wilcox test. Time-dependent ROC curves were analyzed by "ROC" $\mathrm{R}$ package. All statistical tests were two-sided, and $p$-value $<0.05$ was considered statistically significant.

\section{Results}

\section{Clinical Characteristics}

The procedure for the study is shown in the flowchart in detail (Figure 1). Three hundred patients and 348 patients diagnosed with GC were included in the GEO cohort and TCGA cohort, respectively. GEO cohort included 199 (66.33\%) male and 101 (33.67\%) female, and the TCGA cohort included 110 (31.61\%) male and $235(67.53 \%)$ female. Rest detailed clinical characteristics including age, pathologic stage, and TNM stage of all patients of these two cohorts are shown in Tables 1 \& 2. 
Table 1. The characteristics of patients in the GEO cohort

\begin{tabular}{ll}
\hline Variables & Case, $\mathrm{N}(\%)$ \\
\hline Age at diagnosis & \\
$\leq 60$ & $117(39.00 \%)$ \\
$>60$ & $183(61.00 \%)$ \\
Gender & \\
Male & $199(66.33 \%)$ \\
Female & $101(33.67 \%)$ \\
Pathological-Stage & \\
I & $30(10.00 \%)$ \\
II & $96(32.00 \%)$ \\
III & $95(31.67 \%)$ \\
IV & $77(25.67 \%)$ \\
NA & $2(0.67 \%)$ \\
TNM-T & \\
T1 & $0(0.00 \%)$ \\
T2 & $186(62.00 \%)$ \\
T3 & $91(30.33 \%)$ \\
T4 & $21(7.00 \%)$ \\
NA & $2(0.67 \%)$ \\
TNM-N & \\
N0 & $38(12.67 \%)$ \\
N1 & $131(43.67 \%)$ \\
N2 & $80(26.67 \%)$ \\
N3 & $51(17.00 \%)$ \\
TNM-M & \\
M0 & $273(91.00 \%)$ \\
M1 & $27(9.00 \%)$ \\
\hline &
\end{tabular}

\section{Identification of Differentially Expressed Immune Cells between Normal Tissue and Tumor Tissue}

First, we studied the ratio of immune cell composition between tumors and normal tissues in the GEO and TCGA cohorts, respectively. In the GEO cohort, 100 normal and 300 tumor samples were qualified with CIBERSORT $p$-value $<0.05$. The composition of 22 infiltrating immune cells was analyzed. We performed heatmap and barplot analysis of foresaid immune cells to illustrate the differential composition of immune cells in different samples (Figure 2A-B). In Figure 2C, we showed that the formation of many immune cells was significantly different between normal tissue and GC tissue. T cells CD8, $\mathrm{T}$ cells CD4 memory resting, $\mathrm{T}$ cells CD4 memory activated, $\mathrm{T}$ cells follicular helper, $\mathrm{T}$ cells gamma delta, Macrophages M0, Macrophages M1, Dendritic cells resting, Dendritic cells activated, Mast cells activated, Eosinophils and Neutrophils were noticeably increased in GC tissues compared to normal tissues with $p$-value $<0.05$. In the TCGA cohort, 13 normal and 196 tumor samples were qualified with CIBERSORT $p$-value $<0.05$. The same as the GEO cohort, we analyzed the composition of 22 infiltrating immune cells. The composition of the immune cells in normal and tumor tissues was shown in Figure 2D-2E. The fractions of T cells CD4 memory activated, macrophages M0, macrophages M1 and macrophages M2 were significantly higher ( $p$-value $<0.05)$ in GC tissues than in normal tissues, shown in
Figure 2F.

Table 2. The characteristics of patients in the TCGA cohort

\begin{tabular}{ll}
\hline Variables & Case, N $(\%)$ \\
\hline Age at diagnosis & $110(31.61 \%)$ \\
$\leq 60$ & $235(67.53 \%)$ \\
$>60$ & $2(0.57 \%)$ \\
NA & \\
Gender & \\
Male & $218(62.64 \%)$ \\
Female & $129(39.94 \%)$ \\
Pathological-Stage & \\
I & $52(14.94 \%)$ \\
II & $103(19.60 \%)$ \\
III & $135(38.79 \%)$ \\
IV & $35(10.05 \%)$ \\
NA & $22(6.32 \%)$ \\
TNM-T & \\
T1 & $20(5.75 \%)$ \\
T2 & $78(22.41 \%)$ \\
T3 & $156(44.83 \%)$ \\
T4 & $85(24.43 \%)$ \\
NA & $8(2.30 \%)$ \\
TNM-N & \\
N0 & $104(29.89 \%)$ \\
N1 & $91(26.15 \%)$ \\
N2 & $71(20.40 \%)$ \\
N3 & $65(18.68 \%)$ \\
NA & $16(4.60 \%)$ \\
TNM-M & \\
M0 & $307(88.22 \%)$ \\
M1 & $24(6.90 \%)$ \\
NA & $16(4.60 \%)$ \\
\hline &
\end{tabular}

\section{Relationships between Immune Cells in GC Tissues and Correlation of Immune Cells with Clinical Features}

To identify immune cells increased in both the GEO cohort and the TCGA cohort, we took the intersection of immune cells increased in both cohorts. As shown in Figure 3A, three immune cells (T cells CD4 memory activated, Macrophages M0 and Macrophages M1) were found higher in GC tissues than in normal tissues in both GEO and TCGA cohort. The relationships between different types of immune cells were analyzed by the "corrplot" package via Pearson correlation coefficient.

As shown in Figure 3B, T cells CD4 memory activated was positively related with Macrophages M1 and Mast cells resting with Correlation coefficient $>0.5$. $\mathrm{T}$ cells CD4 memory activated was negatively correlated with T cells CD4 memory resting, and Mast cells activated was negatively correlated with Mast cells resting with Correlation coefficient $<-0.5$ in the GEO cohort. In the TCGA cohort (Figure 3C), T cells CD4 memory resting was negatively related with $\mathrm{T}$ cells CD8 and T cells CD4 memory activated with Correlation coefficient -0.49 and -0.47 , respectively, which was partly consistent with the correlation of immune cells in the GEO cohort. 


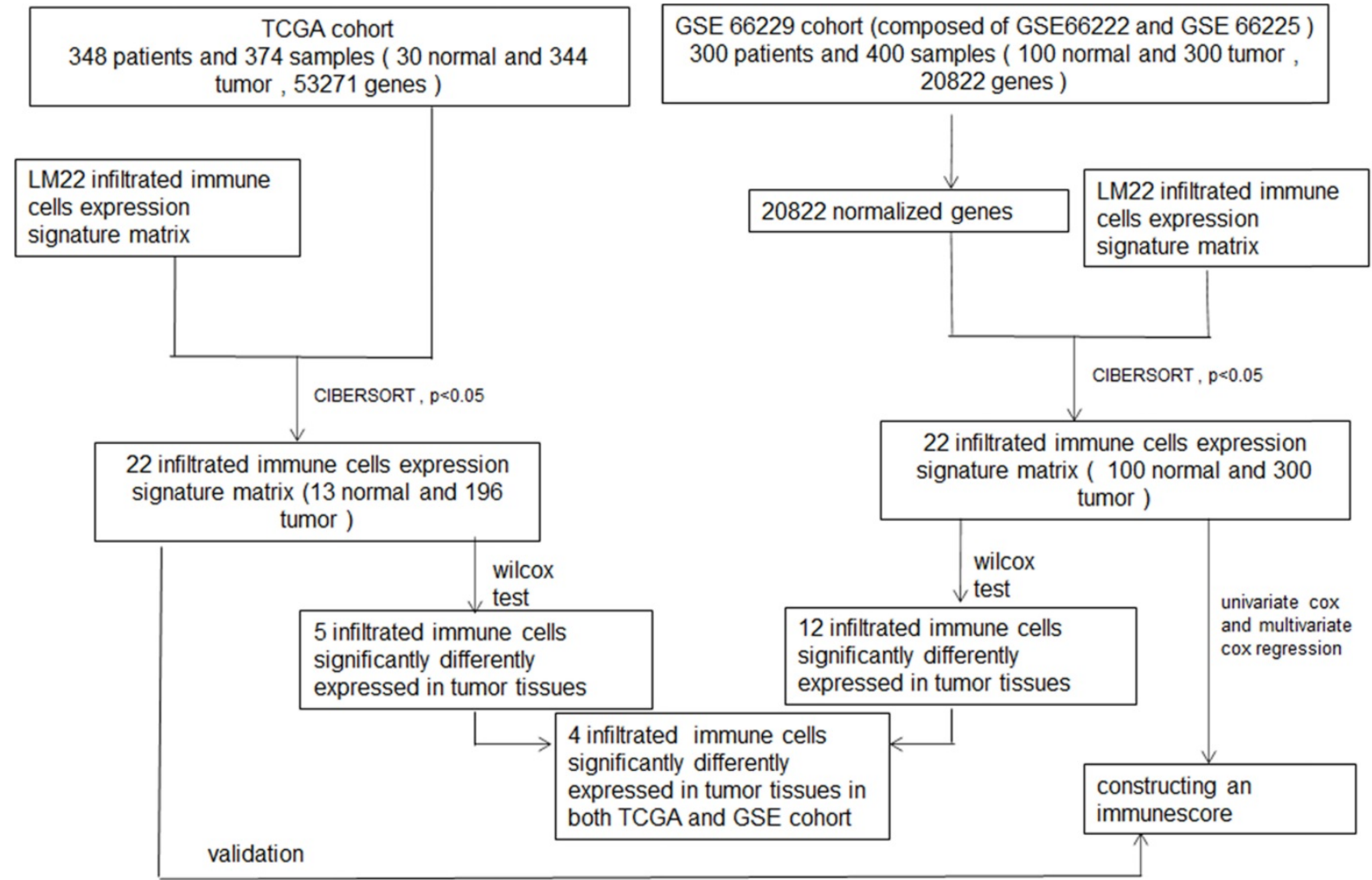

Figure 1. Flowchart of whole procedure including immune cells analysis and their correlations with clinical information, and immune score model construction of immune cells, and validation of the model.
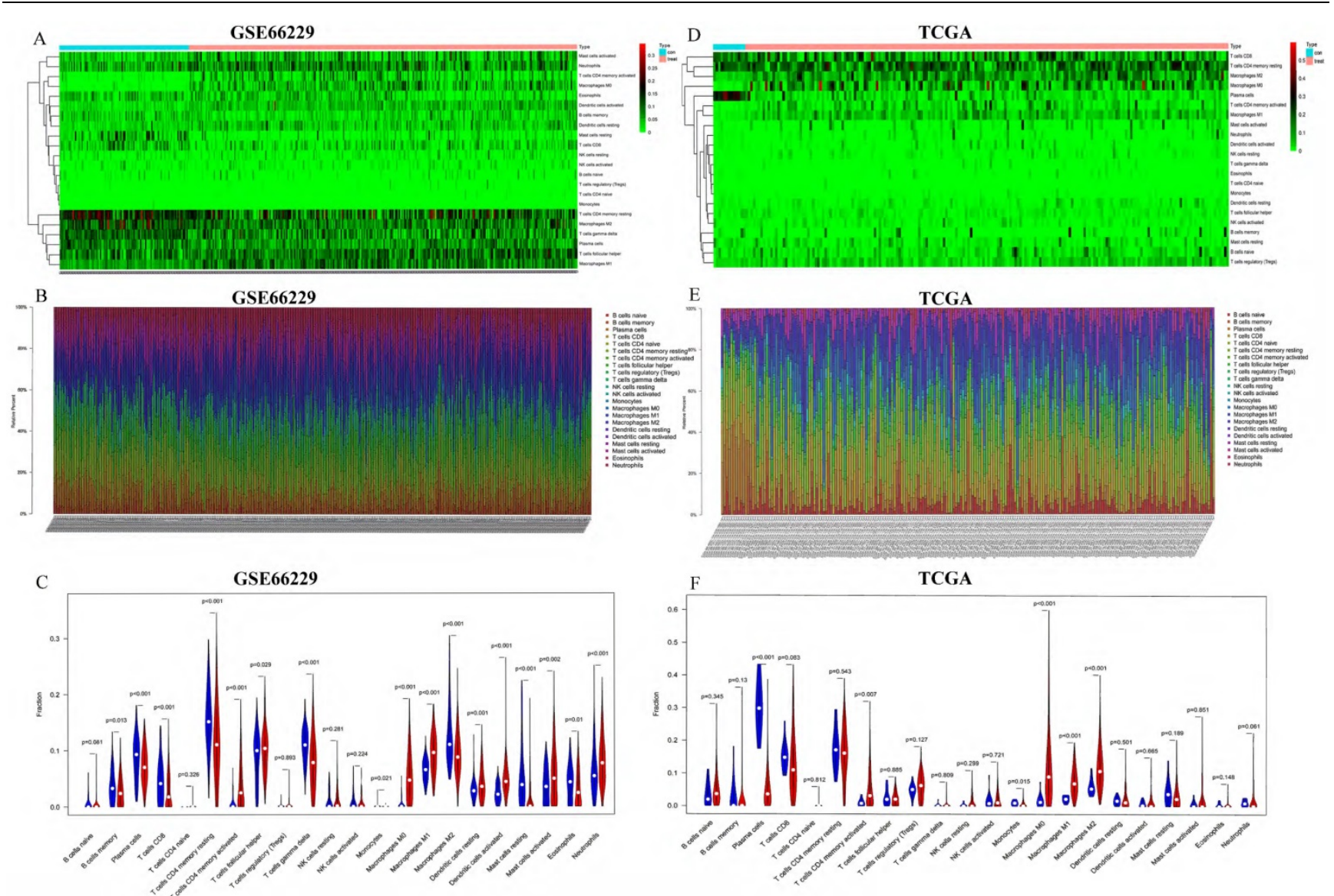

Figure 2. Analysis of TIICs in 100 normal and 300 tumor tissues in the GEO cohort and 13 normal and 196 tumor tissues in the TCGA cohort. (A, D) Heatmaps of TIICs in normal and tumor tissues from the GEO cohort and TCGA cohort, respectively. (B, E) Barplots of TIICs in normal and tumor tissues from the GEO cohort and TCGA cohort. $(\mathbf{C}, \mathbf{F})$ The different fraction of immune cells between normal and tumor tissues. 
A

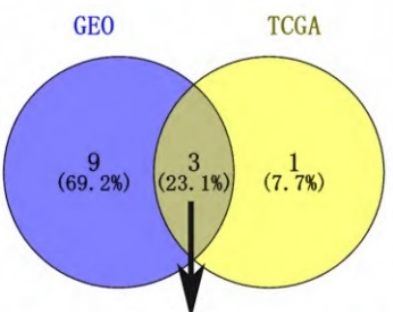

T cells CD4 memory activated Macrophages M0 Macrophages M1

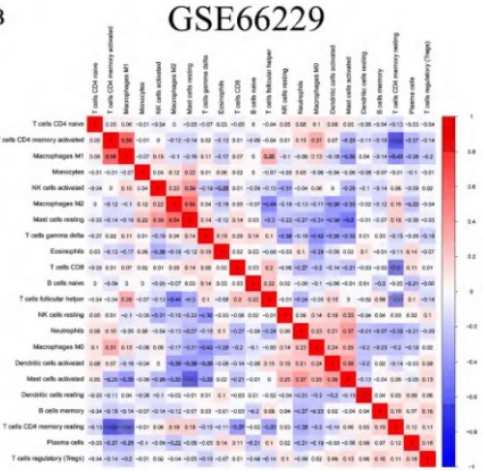

C TCGA

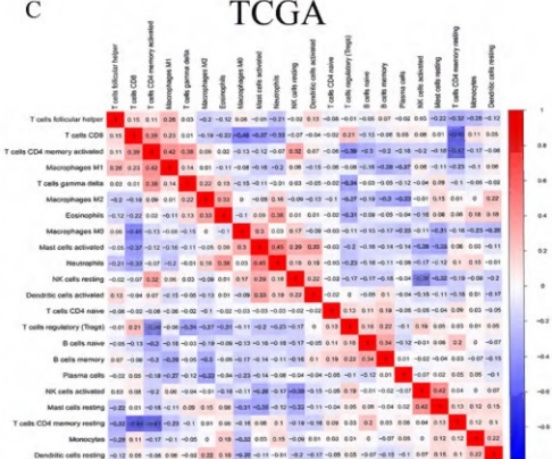

Figure 3. (A) The blue cycle represented immune cells increased in the GEO cohort. The yellow cycle represented immune cells increased in the TCGA cohort. Correlation of immune cells with each other in GEO (B) and TCGA (C).
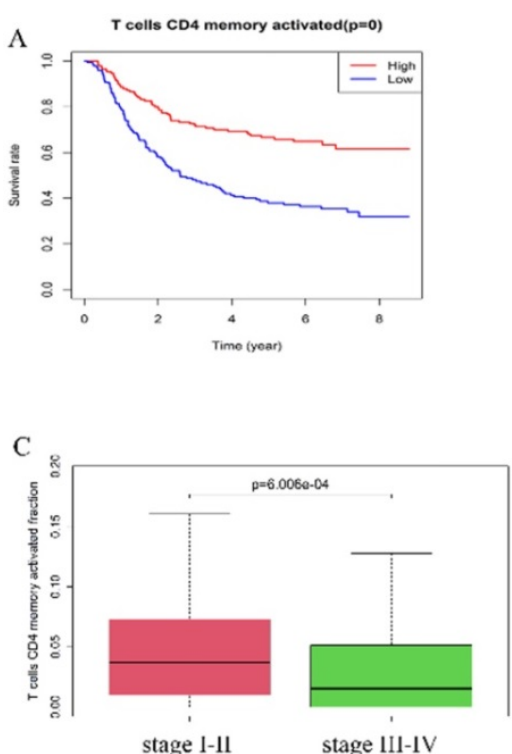

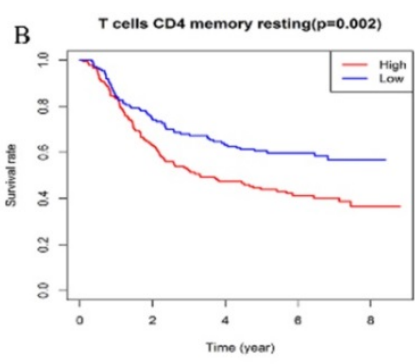

D

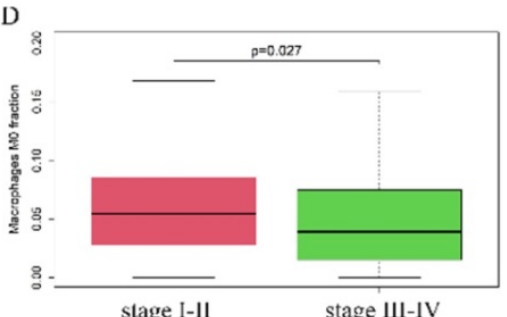

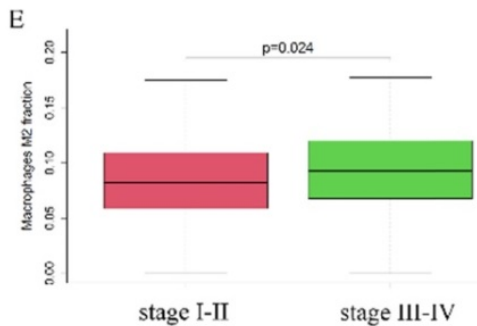

Figure 4. A high fraction of T cells CD4 memory activated $(\mathbf{A})$ and a low fraction of $T$ cells $C D 4$ memory resting (B) was associated with better prognosis. (C) T cells CD4 memory activated and (D) Macrophages M0 were lower in stage III-IV than stage I-II. (E) Macrophages M2 was higher in stage III-IV than stage I-II.

Table 3. Univariate Cox Regression Analysis for 22 Immune Cells in Gastric cancer Patients

\begin{tabular}{lllll}
\hline ID & HR & HR.95L & HR.95H & pvalue \\
\hline T cells CD4 memory activated & 0.000515 & $1.56 \mathrm{E}-05$ & 0.017058 & $2.24 \mathrm{E}-05$ \\
T cells CD4 memory resting & 12.02513 & 2.133142 & 67.78905 & 0.004824 \\
Macrophages M2 & 52.22081 & 2.458491 & 1109.222 & 0.011184 \\
Mast cells activated & 11.88592 & 1.059103 & 133.3913 & 0.044802 \\
Plasma cells & 0.030911 & 0.000776 & 1.231519 & 0.064429 \\
Macrophages M1 & 0.053303 & 0.001711 & 1.6603 & 0.094723 \\
Dendritic cells resting & 11.73588 & 0.205767 & 669.3522 & 0.232616 \\
T cells gamma delta & 4.242797 & 0.333383 & 53.9959 & 0.265462 \\
Neutrophils & 0.214906 & 0.012666 & 3.646489 & 0.287165 \\
Eosinophils & 0.142002 & 0.003614 & 5.579845 & 0.297358 \\
Macrophages M0 & 0.213325 & 0.010996 & 4.138529 & 0.307179 \\
T cells follicular helper & 0.254205 & 0.012217 & 5.289503 & 0.376491 \\
NK cells resting & 0.053226 & $6.20 \mathrm{E}-05$ & 45.67551 & 0.394715 \\
T cells CD4 naive & $1.51 \mathrm{E}-08$ & $1.17 \mathrm{E}-26$ & $1.96 \mathrm{E}+10$ & 0.397427 \\
Dendritic cells activated & 0.243218 & 0.001937 & 30.53197 & 0.566374 \\
T cells regulatory (Tregs) & 0.003249 & $8.44 \mathrm{E}-12$ & 1250128 & 0.569996 \\
B cells naive & 8.782117 & 0.004589 & 16806.77 & 0.573078 \\
NK cells activated & 6.86765 & 0.005365 & 8791.848 & 0.597617 \\
Mast cells resting & 1.576252 & 0.013688 & 181.5206 & 0.850947 \\
T cells CD8 & 0.743425 & 0.020419 & 27.0669 & 0.87158 \\
B cells memory & 0.874211 & 0.010045 & 76.08398 & 0.952957 \\
Monocytes & 0 & 0 & Inf & 0.994677 \\
\hline
\end{tabular}

Furthermore, we explored the correlation of immune cells with the overall survival and pathologic stage. In the GEO cohort, high fraction of T cells CD4 memory activated ( $p$-value $<0.01)$ was associated with better survival rate (Figure 4A), while a high fraction of $\mathrm{T}$ cells memory resting $(p=0.002)$ was a risk factor (Figure 4B). The fraction of $\mathrm{T}$ cells CD4 memory activated $(P<0.001)$ and Macrophages M0 $(p=0.027)$ was higher in stage I-II tumors compared with stage III-IV tumors while the fraction of Macrophages M2 $(p=0.024)$ was higher in stage III-IV tumors than in stage I-II tumors (Figure 4C-E).

\section{Construction and Validation of the Immune Risk Score Model}

Considering the number of samples, we chose the GEO cohort as the training set. Univariate Cox regression was applied to identify immune cells associated with the prognosis of GC patients. As shown in Table 3, T cells CD4 memory activated, T cells CD4 memory resting, Macrophages M2, and 
Mast cells activated were found associated with gastric cancer survival risk significantly with $p$-value $<0.05$. Moreover, these four immune cells were included for multivariate Cox regression analysis to construct an immune risk score model (Figure 5A) in which we could see that a high fraction of T cell CD4 memory activated was a potential protective factor. The risk score calculation formula was: risk score= $(0.5500$ * fraction of $\mathrm{T}$ cells CD4 memory resting $)+$ $(-8.8257 *$ fraction of $\mathrm{T}$ cells CD4 memory activated $)+$ $(5.8963$ * fraction of Macrophages M2) + (2.5130 * fraction of Mast cells activated). The ROC curve showed that this model had a passable sensitivity and specificity in predicting overall survival. The AUC of one-year, three-year, and five-year survival was 0.64, 0.67, and 0.67, respectively (Figure 5B). Moreover, we calculated the risk score of each patient through this model, and the KM survival curve indicated that patients with high-risk scores had poor prognosis

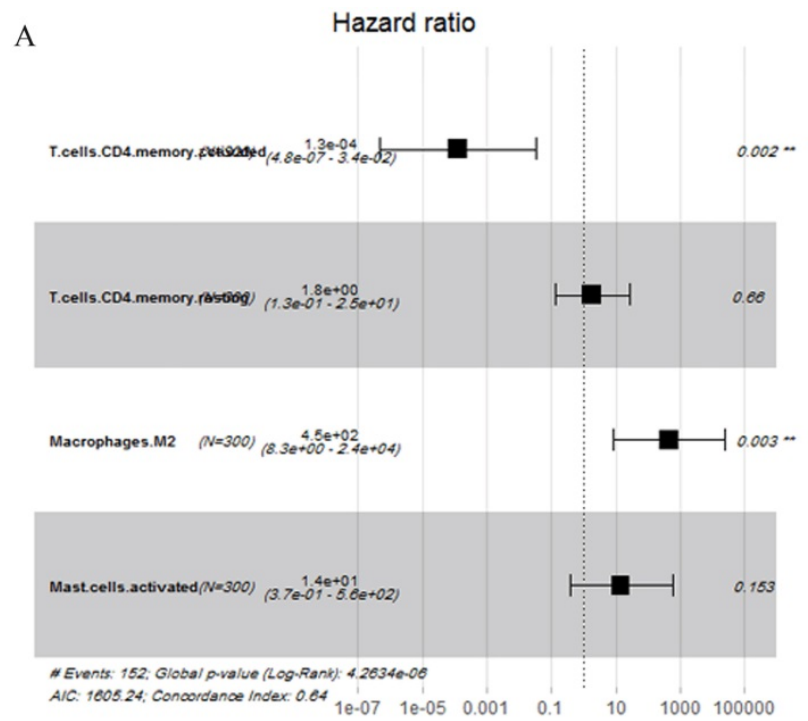

C

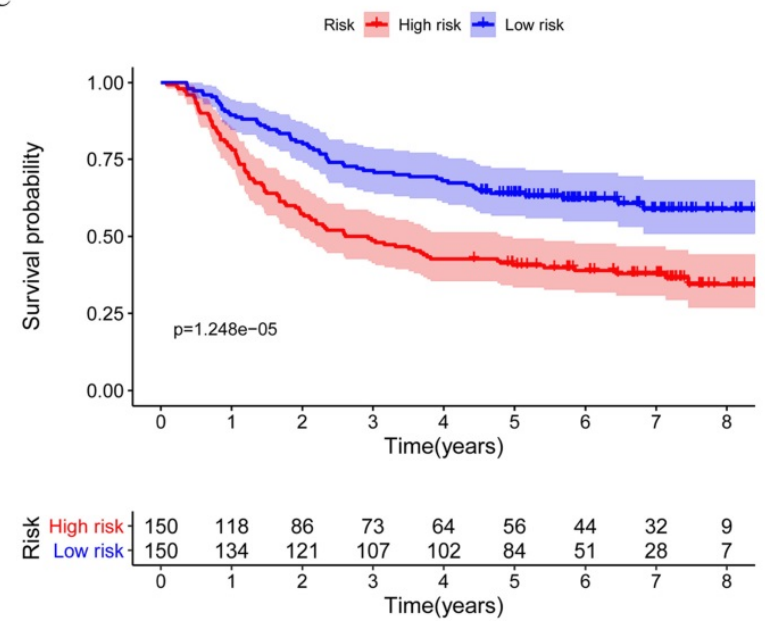

compared with patients with low-risk scores (Figure 5C). We ranked GC patients according to risk score (Figure 5D), and patients' status was presented in Figure 5E. We found that death occurred more in the high-risk group than in the low-risk group, which was consistent with our previous result.

To test the applicability of our model, we applied the model in the TCGA cohort for validation. Each patient in the TCGA cohort was assigned a risk score according to the risk score model. As shown in Figure 6A, this model still had a passable sensitivity and specificity in predicting overall survival. Low-risk patients had significantly better survival probability than high-risk patients, which was indicated by the KM survival plot (Figure 6B). The distribution of risk score and patients' survival status was presented in Figure 6C-D. Consistent with the training cohort, more deaths occurred in the high-risk group than in the low-risk group.

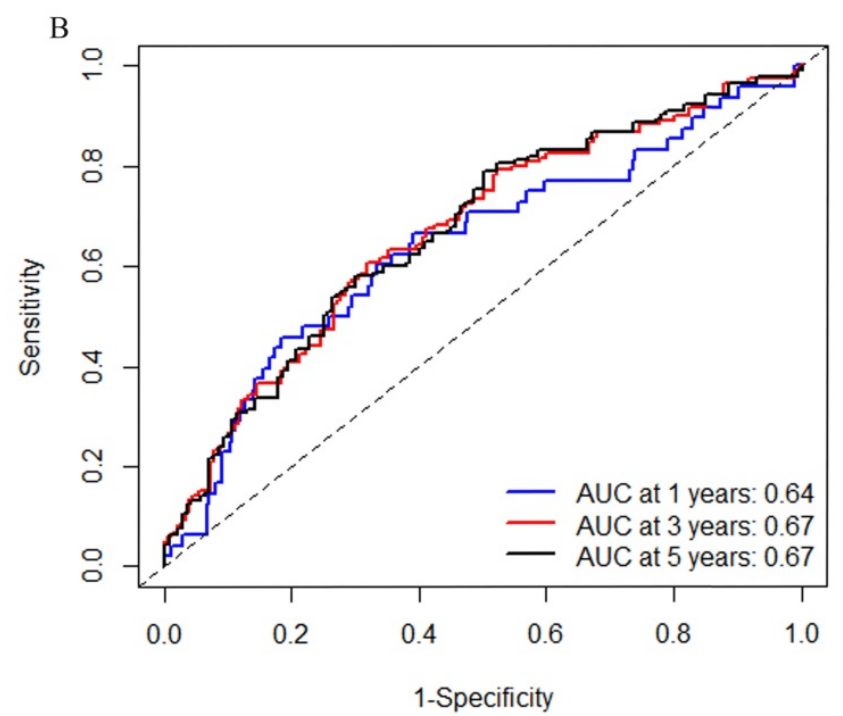

D

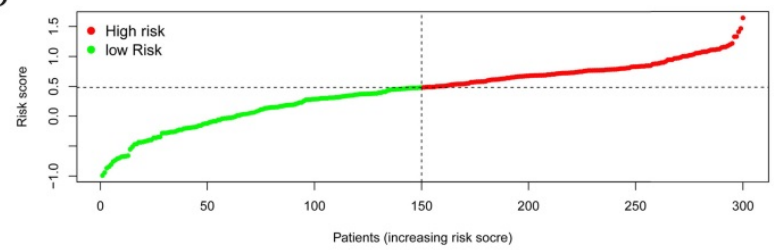

E

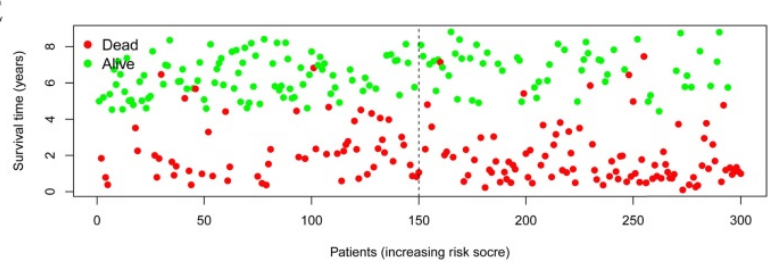

Figure 5. Construction of the immune risk score model. (A) T cells CD4 memory activated, T cells CD4 memory resting, Macrophages M2, and Mast cells activated were selected to construct an immune risk score model through multivariate cox regression in the GEO cohort. (B) ROC curve analysis of prognosis prediction by the model. (C) KM survival curve indicted that high-risk scores were associated with poor prognosis. (D) Distribution of the immune score of GC patients. (E) Distribution of patients' status. 

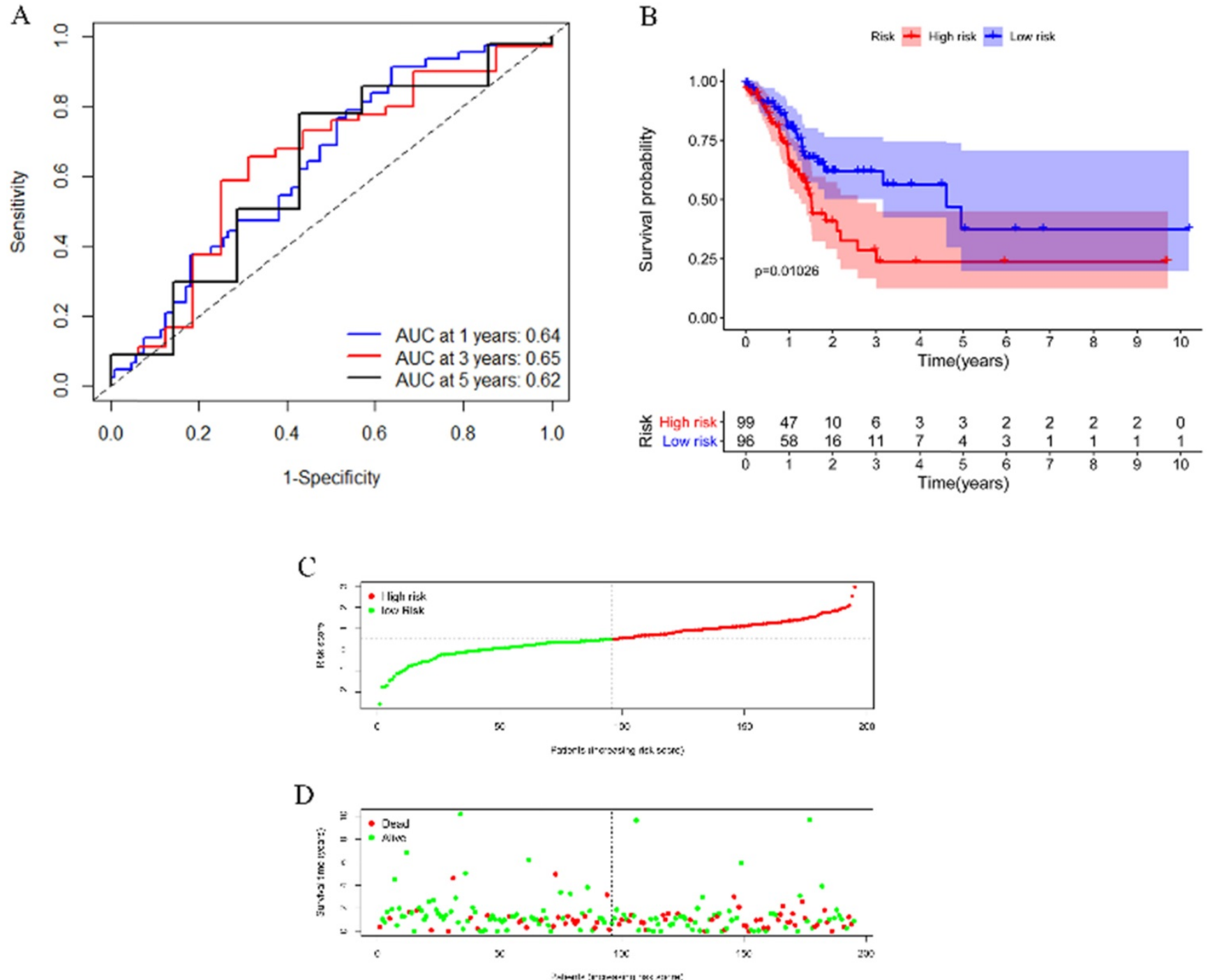

Figure 6. Validation of the model by the TCGA cohort. (A) ROC curve analysis of survival prediction by the model. (B) KM survival curve revealed that patients with high risk had a poor prognosis. (C) Distribution of the immune score of GC patients. (D) Distribution of status of GC patients.

\section{The Immune Risk Score Model could predict the Prognosis of Gastric Cancer Independently}

To determine whether the immune risk score model was independent of clinical parameters, we performed univariate cox and multivariate cox regression. In the GEO cohort (Figure 7A), high-risk score, T, N, M, and pathologic stage were related to poor prognosis while age and high-risk score were associated with poor prognosis in the TCGA cohort (Figure 7B). To identify factors that can predict prognosis independently, we applied multivariate Cox regression. As shown in Figure 7C, stages, TNM-M stage, age, and risk score were independent factors with $p$-value $<0.05$ in the GEO cohort, while in the TCGA cohort, only risk score could predict overall survival independently (Figure 7D). It proved that our model was an independent and reliable factor for estimating the prognosis of Gastric cancer.
Furthermore, the risk score model was validated within patients subgroups divided by age, gender, stage, and T-stages, respectively, in the training cohort. Similarly, high-risk patients had poorer outcome compared with low-risk patients in the male $(P<001)$, age $<60(P<0.01)$, age $\geq 60(P<0.001)$, stage I-II $(p=0.01859)$, stage III-IV $(P<0.001)$, TI-II $(P<0.001)$, and TIII-IV $(p=0.02526)$ subgroups (Figure 8A, 8C-H). However, the high-risk patients in the female subgroup $(p=0.05682)$ didn't significantly differ with low-risk patients in survival rate (Figure $8 \mathbf{8 B}$ ). Together, these results indicated the reliability of our model.

\section{Discussion}

Gastric cancer is a common cause of tumorrelated death. Due to the heterogeneity of Gastric cancer, patients even with the same pathologic stage and TNM stage responded differently to similar 
therapy. Recently, immunotherapy based on blockade of immune checkpoints has obtained appreciable efficacy in GC. The infiltrating immune cells, especially $\mathrm{T}$ cells in tumors, play a vital role in recognizing and eradicating cancer cells, and cancer cells use multiple signaling pathways to inhibit the activity of $\mathrm{T}$ cell, thus suppressing tumor immunity [15]. The therapy blocking PD-1/PD-L1 exerted a favorable response rate by reactivating the effector activity of infiltrating $T$ cells in many cancers $[16,17]$. However, the cancer cells inhibit immune cells function by multiple mechanisms [18] for which immunotherapy does not suit each patient. Therefore, the identification of novel biomarkers is vital for stratifying patients and choosing appropriate therapy. Based on this purpose, we analyzed 22 infiltrating immune cells in Gastric cancer and constructed an immune risk score model using immune cells to predict overall survival in this study.

We performed the CIBERSORT algorithm to evaluate the composition fraction of immune cells in gastric cancer tissues and normal tissues. T cells CD4 memory activated, macrophages $\mathrm{M} 0$, and macrophages M1 were three immune cells increased in tumor tissues compared with normal tissues in both GEO and TCGA cohorts. Macrophages are one of the
A

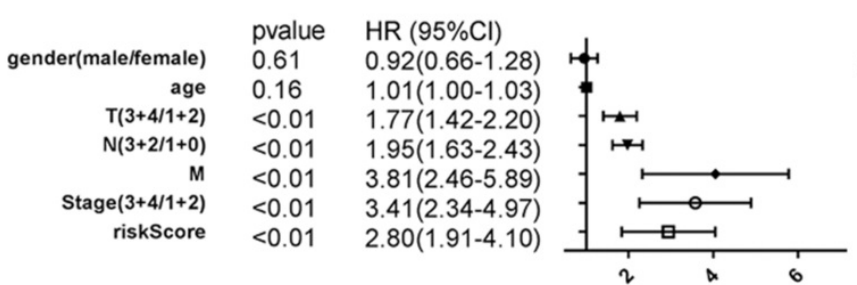

B

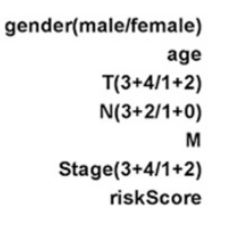

TCGA

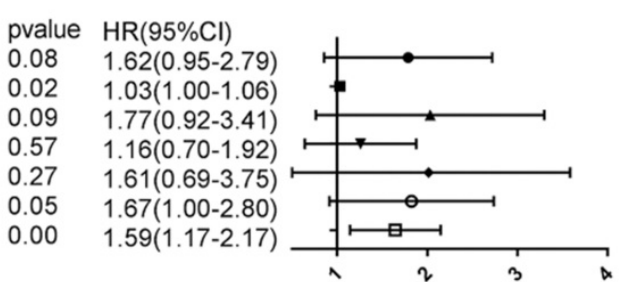

$\mathrm{C}$

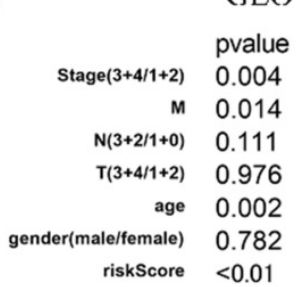

GEO

$\mathrm{HR}(95 \% \mathrm{Cl})$

1.72( $1.19 \sim 2.49)$

$1.90(1.14 \sim 3.15)$

$1.25(0.95 \sim 1.66)$

$0.99(0.74 \sim 1.34)$

$1.72(1.21-2.43)$

$1.05(0.74 \sim 1.49)$

$2.35(1.63 \sim 3.40)$

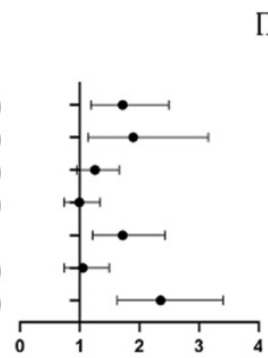

D

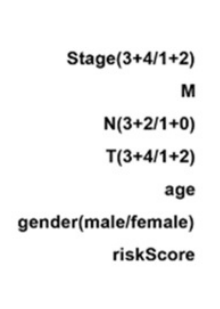

\section{TCGA}

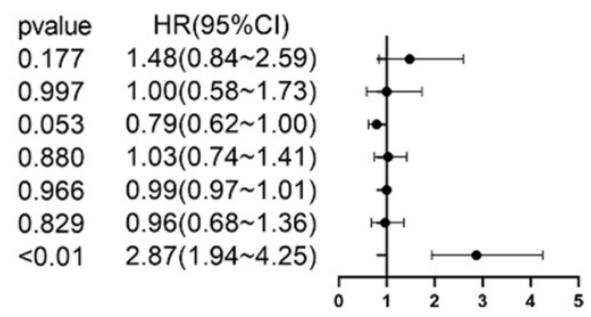

Figure 7. COX regression of clinical factors might affect prognosis. Univariate cox regression of seven factors in the GEO cohort (A) and TCGA cohort (B). multivariate cox regression of seven factors in the GEO cohort (C) and TCGA cohort (D).
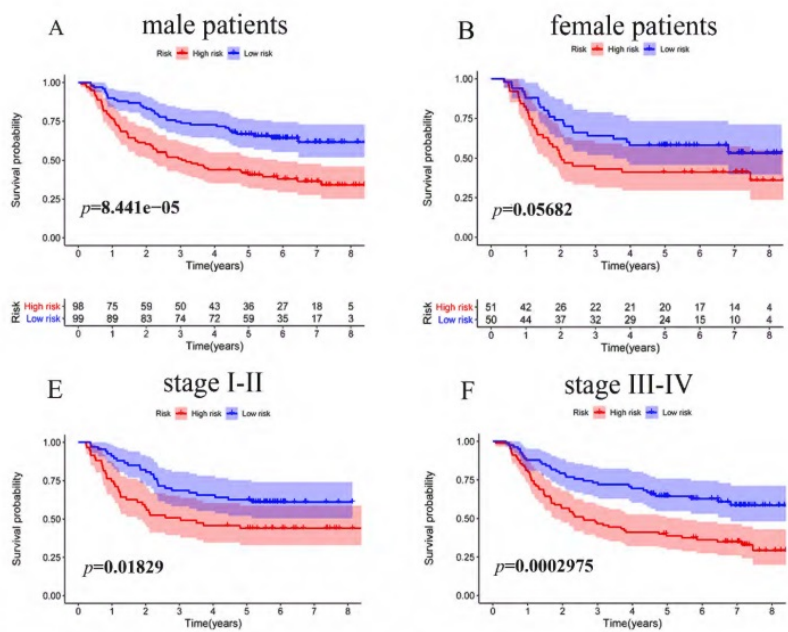

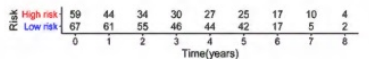

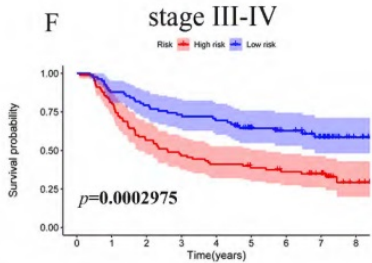

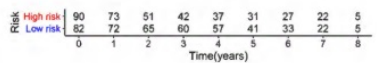
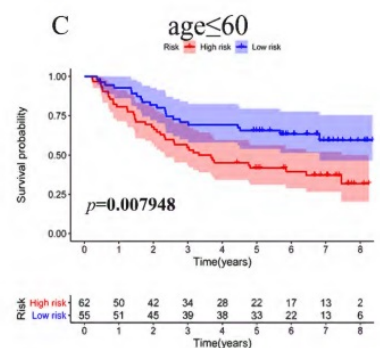

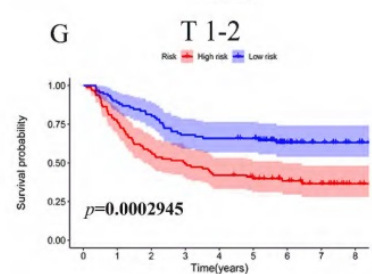

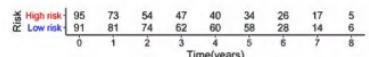
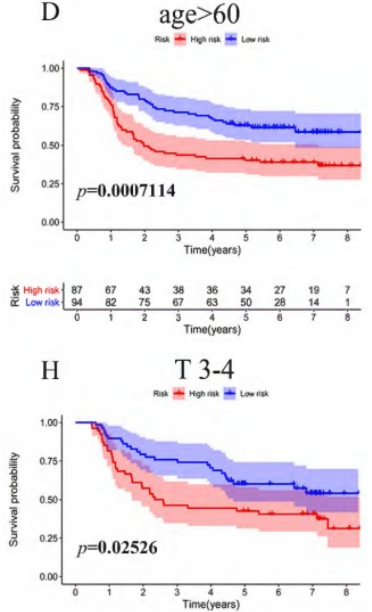

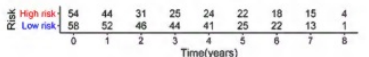

Figure 8. KM survival analysis of different patient subgroups in the GEO cohort. (A) male patients, (B) female patients, (C) patients with age $\leq 60$, (D) patients with age $>60$, (E) patients with stage I-II GC, (F) patients with stage III-IV, $(\mathbf{G})$ patients with T I-II, and $(\mathbf{H})$ patients with T III-IV. 
primary immune cells in tumor immune microenvironment and can function differently in response to microenvironmental signals [19, 20]. Macrophages are versatile cells that can be polarized into pro-inflammatory macrophages M1 and immunosuppressive macrophages M2 under different chemokines stimulation [21]. Both M1 and M2 participate in the tumor progression process, but M1 is a mainly protective factor by activating the production of toxic intermediates and reactive oxygen intermediates, while $\mathrm{M} 2$ is a risk factor for it promotes tumor progression and metastasis [22, 23]. Macrophages M0 has similar but slightly weaker functions as M1. In our analysis, Macrophages had no significant relationship with overall survival, but significantly correlated with pathologic stages. In the GEO cohort, the correlations of Macrophages and stages were consistent with foresaid studies.

In our survival analysis, a high fraction of $\mathrm{T}$ cells CD4 memory activated was related to high survival possibility while a high fraction of $\mathrm{T}$ cells $\mathrm{CD} 4$ memory resting was associated with poor survival probability. $T$ cells are able to recognize and eradicate cancer cells through multiple steps [15]. It has been reported that polyfunctional CD8+ effector cytotoxic $\mathrm{T}$ cells (CTL) in vivo are critical for anti-tumor immunity [24]. A study has shown that with the help of CD4 T cells, CTL polyfunctionality and anti-tumor effect both increased [25]. Moreover, CD4 memory T cells display multifunctional and resistant cytokine production in response to stimulation [26]. A study about bladder cancer also indicated that a high fraction of $\mathrm{T}$ cells CD4 memory activated was associated with better outcomes [27]. Cancer cells can not only activate $\mathrm{T}$ cell responses but can also inhibit the activity of $\mathrm{T}$ cells through overexpression of checkpoint molecules, which is called immune tolerance [15]. Besides, tumor cells can adjust the tumor immune microenvironment to facilitate the growth of tumor cells by expanding regulatory T-cells (Tregs) and other immune cells-regulatory cells [28, 29]. Normally, the resting formation of immune cells is associated with the dormant immune microenvironment and poor prognosis. The current immunotherapies aimed to reactivate dormant antitumor immunity by targeting checkpoint molecules have achieved considerable progress [30]. These results suggested that $\mathrm{T}$ cells CD4 memory activated could restrict the growth of cancer cells. In contrast, $\mathrm{T}$ cells CD4 memory resting might associate with poor prognosis partly because it correlates with immune tolerance.

Furthermore, univariate cox and multivariate cox regression were performed to construct an immune risk model using the 22 infiltrating immune cells. KM survival curve suggested that patients with high-risk scores had significantly poor survival rates than patients with low-risk scores in both TCGA and GEO cohorts $(P<0.001$ and $p=0.01026$, respectively). ROC curve demonstrated the reliability of the model in predicting overall survival (AUC at three years was 0.67 and 0.65 in the GEO cohort and the TCGA cohort, respectively). We also performed multivariate cox regression to validate our model as an independent factor for predicting overall survival of gastric cancer. Currently, immunotherapies based on blockade of immune checkpoints (such as CTLA-4 [31], PD1, and PD-L1) receptors and their ligands have made considerable progress not only in Gastric cancer but also in colon, lung, and renal carcinoma.[16] However, effective biomarkers for prognosis predicting and selecting patients sensitive to immunotherapy and can benefit from the therapy the best remains unclear. The efficacy of immunotherapy largely depends on the response of infiltrating immune cells in the tumor immune microenvironment $[17,18]$. Hence, discovering an immune biomarker for predicting prognosis and choosing therapy is of great importance.

The role of infiltrating immune cells in gastric cancer has been explored in several previous studies. Liu $\mathrm{K}$ et al. explored the distribution and density of tumor-infiltrating immune cells in gastric cancer through immunostaining and indicated that immune cells were significantly associated with the prognosis of GC patients [32]. Wang JT et al. revealed that IL17A+ cells infiltration improved anti-tumor contexture and response to immunotherapy in GC [33]. Li L et al. constructed an immune score model by tumor-infiltrating immune cells; however, they only validated the nomogram model instead of the score model by external datasets [34]. In our study, we constructed an immune score model with reliability and validated it with an external dataset, which is our strength. Some studies investigated the relationship between immune checkpoint molecules and immune cells and their correlation with overall survival. Wang et al. reported that PD-L1 expression and CD8 T cell infiltration were associated with better outcomes in advanced GC [35]. Wang $M$ et al. identified SUPV3L1 and SLC22A17 as two genes that could affect the immune cells and prognosis in GC [36]. Zhou Y et al. found that Fatty acid synthase (FASN) was associated with immune cells and the prognosis of Gastric cancer patients [37]. All these studies provided a new comprehension of how Gastric cancer progressed and potential therapies targets. However, the shortcoming of these studies is that they focused on only one gene or one cell and neglected that tumors comprise complex biological processes and varieties of cells. It 
should be noted that we considered four immune cells when constructing the immune risk score model. Besides, the model was constructed in the GEO cohort in which data was from Asian people but validated in the TCGA cohort in which data was from people without specific ethnicities. Therefore, we thought our model might be able to extrapolate to different races. There certainly are limitations to our study. The major limitation of our study is that we didn't validate our results by experiments or our own clinical specimens.

\section{Conclusion}

In summary, the fraction of immune cells is correlated with survival in GC. Our model is a reliable and independent factor for predicting prognosis. With the development of sequencing technology, we think our model has great potential in clinical practice. The model may play an essential role in patient stratification and therapy.

\section{Acknowledgements}

This study was supported by the National Science Foundation of China (No: 81870390; No: 81800494).

\section{Author contributions}

Wenjie Li and Qiu Zhao designed the study. Wenjie Li, Mengting Li, and Yannan Peng collected the data. Haizhou Wang and Yuanyuan Lu analyzed the data. Shouquan Dong, Fei Xu, and Fan Wang supervised the data and provided statistical advice. Wenjie Li and Mengting Li wrote the paper, Qiu Zhao and Lan Liu reviewed the paper. All authors approved the final manuscript.

\section{Competing Interests}

The authors have declared that no competing interest exists.

\section{References}

1. Bray F, Ferlay J, Soerjomataram I, Siegel RL, Torre LA, Jemal A. Global cancer statistics 2018: GLOBOCAN estimates of incidence and mortality worldwide for 36 cancers in 185 countries. CA: a cancer journal for clinicians. 2018; 68: 394-424.

2. Shah MA, Ajani JA. Gastric cancer--an enigmatic and heterogeneous disease. JAMA. 2010; 303: 1753-4.

3. Noh SH, Park SR, Yang HK, Chung HC, Chung IJ, Kim SW, et al. Adjuvant capecitabine plus oxaliplatin for gastric cancer after D2 gastrectomy (CLASSIC): 5-year follow-up of an open-label, randomised phase 3 trial. The Lancet Oncology. 2014; 15: 1389-96.

4. Ajani JA, D'Amico TA, Almhanna K, Bentrem DJ, Chao J, Das P, et al. Gastric Cancer, Version 3.2016, NCCN Clinical Practice Guidelines in Oncology. Journal of the National Comprehensive Cancer Network : JNCCN. 2016; 14: 1286-312.

5. Hanahan D, Weinberg RA. Hallmarks of cancer: the next generation. Cell. 2011; 144: 646-74.

6. Becht E, Giraldo NA, Germain C, de Reyniès A, Laurent-Puig P, Zucman-Rossi $\mathrm{J}$, et al. Immune Contexture, Immunoscore, and Malignant Cell Molecular Subgroups for Prognostic and Theranostic Classifications of Cancers. Advances in immunology. 2016; 130: 95-190.

7. Galon I, Costes A, Sanchez-Cabo F, Kirilovsky A, Mlecnik B, Lagorce-Pagès C, et al. Type, density, and location of immune cells within human colorectal tumors predict clinical outcome. Science (New York, NY). 2006; 313: 1960-4
8. Angell H, Galon J. From the immune contexture to the Immunoscore: the role of prognostic and predictive immune markers in cancer. Current opinion in immunology. 2013; 25: 261-7.

9. Song C, Phuengkham H, Kim YS, Dinh VV, Lee I, Shin IW, et al. Syringeable immunotherapeutic nanogel reshapes tumor microenvironment and prevents tumor metastasis and recurrence. Nature communications. 2019; 10: 3745.

10. Wang M, Huang YK, Kong JC, Sun Y, Tantalo DG, Yeang HXA, et al. High-dimensional analyses reveal a distinct role of T-cell subsets in the immune microenvironment of gastric cancer. Clinical \& translational immunology. 2020; 9: e1127.

11. Du Y, Wei Y. Therapeutic Potential of Natural Killer Cells in Gastric Cancer. Frontiers in immunology. 2018; 9: 3095.

12. Wu Y, Wang L, Zhang Y. Dendritic cells as vectors for immunotherapy of tumor and its application for gastric cancer therapy. Cellular \& molecular immunology. 2004; 1: 351-6.

13. Newman AM, Liu CL, Green MR, Gentles AJ, Feng W, Xu Y, et al. Robust enumeration of cell subsets from tissue expression profiles. Nature methods. 2015; 12: 453-7.

14. Friedman J, Hastie T, Tibshirani R. Regularization Paths for Generalized Linear Models via Coordinate Descent. Journal of statistical software. 2010; 33: 1-22.

15. Chen DS, Mellman I. Oncology meets immunology: the cancer-immunity cycle. Immunity. 2013; 39: 1-10.

16. Wu X, Gu Z, Chen Y, Chen B, Chen W, Weng L, et al. Application of PD-1 Blockade in Cancer Immunotherapy. Computational and structural biotechnology journal. 2019; 17: 661-74.

17. Teng MW, Ngiow SF, Ribas A, Smyth MJ. Classifying Cancers Based on T-cell Infiltration and PD-L1. Cancer research. 2015; 75: 2139-45.

18. Motz GT, Coukos G. Deciphering and reversing tumor immune suppression. Immunity. 2013; 39: 61-73.

19. Mantovani A, Sica A, Sozzani S, Allavena P, Vecchi A, Locati M. The chemokine system in diverse forms of macrophage activation and polarization. Trends in immunology. 2004; 25: 677-86.

20. Wiktor-Jedrzejczak W, Gordon S. Cytokine regulation of the macrophage (M phi) system studied using the colony stimulating factor-1-deficient op/op mouse. Physiological reviews. 1996; 76: 927-47.

21. Murray PJ, Wynn TA. Protective and pathogenic functions of macrophage subsets. Nature reviews Immunology. 2011; 11: 723-37.

22. Mantovani A, Sozzani S, Locati M, Allavena P, Sica A. Macrophage polarization: tumor-associated macrophages as a paradigm for polarized M2 mononuclear phagocytes. Trends in immunology. 2002; 23: 549-55.

23. Zhang Y, Meng W, Yue P, Li X. M2 macrophage-derived extracellular vesicles promote gastric cancer progression via a microRNA-130b-3p/MLL3/GRHL2 signaling cascade. Journal of experimental \& clinical cancer research : CR. 2020; 39: 134.

24. Imai N, Ikeda H, Tawara I, Shiku H. Tumor progression inhibits the induction of multifunctionality in adoptively transferred tumor-specific CD8+ T cells. European journal of immunology. 2009; 39: 241-53.

25. Imai N, Tawara I, Yamane M, Muraoka D, Shiku H, Ikeda H. CD4 T cells support polyfunctionality of cytotoxic CD8 T cells with memory potential in immunological control of tumor. Cancer science. 2020; 111: 1958-68.

26. Westerhof LM, McGuire K, MacLellan L, Flynn A, Gray JI, Thomas M, et al. Multifunctional cytokine production reveals functional superiority of memory CD4 T cells. European journal of immunology. 2019; 49: 2019-29.

27. Li W, Zeng J, Luo B, Mao $Y$, Liang $Y$, Zhao $W$, et al. [High expression of activated CD4 memory $\mathrm{T}$ cells and CD8 T cells and low expression of M0 macrophage are associated with better clinical prognosis in bladder cancer patients]. Xi bao yu fen zi mian yi xue za zhi $=$ Chinese journal of cellular and molecular immunology. 2020; 36: 97-103.

28. Francisco LM, Sage PT, Sharpe AH. The PD-1 pathway in tolerance and autoimmunity. Immunological reviews. 2010; 236: 219-42.

29. Pleyer L, Valent P, Greil R. Mesenchymal Stem and Progenitor Cells in Normal and Dysplastic Hematopoiesis-Masters of Survival and Clonality? International journal of molecular sciences. 2016; 17.

30. Greil R, Hutterer E, Hartmann TN, Pleyer L. Reactivation of dormant anti-tumor immunity - a clinical perspective of therapeutic immune checkpoint modulation. Cell communication and signaling: CCS. 2017; 15: 5.

31. Pardoll DM. The blockade of immune checkpoints in cancer immunotherapy. Nature reviews Cancer. 2012; 12: 252-64.

32. Liu K, Yang K, Wu B, Chen H, Chen X, Chen X, et al. Tumor-Infiltrating Immune Cells Are Associated With Prognosis of Gastric Cancer. Medicine. 2015; 94: e1631.

33. Wang JT, Li H, Zhang H, Chen YF, Cao YF, Li RC, et al. Intratumoral IL17-producing cells infiltration correlate with anti-tumor immune contexture and improved response to adjuvant chemotherapy in gastric cancer. Annals of oncology : official journal of the European Society for Medical Oncology. 2019; 30: $266-73$

34. Li L, Ouyang Y, Wang W, Hou D, Zhu Y. The landscape and prognostic value of tumor-infiltrating immune cells in gastric cancer. PeerJ. 2019; 7: e7993.

35. Wang Y, Zhu C, Song W, Li J, Zhao G, Cao H. PD-L1 Expression and CD8 T Cell Infiltration Predict a Favorable Prognosis in Advanced Gastric Cancer. Journal of immunology research. 2018; 2018: 4180517.

36. Wang $\mathrm{M}$, Li Z, Peng $\mathrm{Y}$, Fang J, Fang $\mathrm{T}$, Wu J, et al. Identification of immune cells and mRNA associated with prognosis of gastric cancer. BMC cancer. 2020; 20: 206. 
37. Zhou Y, Su W, Liu H, Chen T, Höti N, Pei H, et al. Fatty acid synthase is a prognostic marker and associated with immune infiltrating in gastric cancers precision medicine. Biomarkers in medicine. 2020; 14: 185-99. 